\title{
LOOP ALGEBRAS, GAUGE INVARIANTS AND A NEW COMPLETELY INTEGRABLE SYSTEM
}

\section{Quinn And S.F. Singer}

One fruitful motivating principle of much research on the family of integrable systems known as "Toda lattices" has been the heuristic assumption that the periodic Toda lattice in an affine Lie algebra is directly analogous to the nonperiodic Toda lattice in a finite-dimensional Lie algebra. This paper shows that the analogy is not perfect. A discrepancy arises because the natural generalization of the structure theory of finite-dimensional simple Lie algebras is not the structure theory of loop algebras but the structure theory of affine Kac-Moody algebras. In this paper we use this natural generalization to construct the natural analog of the nonperiodic Toda lattice. Surprisingly, the result is not the periodic Toda lattice but a new completely integrable system on the periodic Toda lattice phase space. This integrable system is prescribed purely in terms of Lie-theoretic data. The commuting functions are precisely the gauge-invariant functions one obtains by viewing elements of the loop algebra as connections on a bundle over $S^{1}$.

\section{Introduction.}

Toda lattice models belong to a general class of integrable systems associated to vector space splittings of Lie algebras. Suppose that a Lie algebra $\mathfrak{g}$ (with corresponding connected Lie group $\mathbf{G}$ ) splits as a vector space into $\mathfrak{g}=\mathfrak{k}+\mathfrak{b}$, where $\mathfrak{k}$ and $\mathfrak{b}$ are subalgebras (corresponding to subgroups $\mathbf{K}$ and $\mathbf{B}$ respectively). One can naturally identify $\mathfrak{g}^{*} \cong \mathfrak{k}^{*}+\mathfrak{b}^{*}$, and hence coadjoint orbits of $\mathbf{B}$ can be thought of as sitting inside $\mathfrak{g}^{*}$. The invariant functions on $\mathfrak{g}^{*}$, when restricted to a coadjoint orbit of $\mathbf{B}$, give a family of Poissoncommuting functions on that orbit and generate flows there described by Lax pair equations; this is the content of the Kostant-Symes involution theorem $[\mathbf{K o}],[\mathbf{S y}]$. And so, provided the dimensions are right, one can generate interesting completely integrable systems on the coadjoint orbits of $\mathbf{B}$. The classical Toda lattices arise in this way from various splittings of $\operatorname{sl}(n, \mathbb{R})$ and $\operatorname{sl}(n, \mathbb{C})$; their construction generalizes to arbitrary simple Lie algebras (see for instance $[\mathrm{Pe}]$ ). 
In the present paper the algebra considered is a central extension $\widetilde{\mathrm{Lg}}$ of an infinite dimensional loop algebra associated to a finite-dimensional simple Lie algebra $\mathfrak{g}$. In Section 2 we review a well-known construction (e.g. [GW2], [RS], [RSF]) of the real symmetric periodic Toda phase space as a coadjoint orbit, obtained from a splitting of $\widetilde{\mathrm{Lg}}$ in the manner described above. Because of the remarkable fact that coadjoint orbits in $(\widetilde{\mathrm{Lg}})^{*}$ are parameterized by conjugacy classes in the finite dimensional group $\mathbf{G}$, a natural choice of invariant functions on $(\widetilde{\mathrm{Lg}})^{*}$ is available via the class functions of $\mathbf{G}$. We give a construction of these invariant functions in Section 3, and in Section 4 address a technical issue that ensures the generic non-degeneracy of these functions on the Toda phase space. In Section 5 we show that this family of functions generates a completely integrable system on our phase space and that the Hamiltonian of the periodic Toda lattice is not contained in this family. Finally, we have included a glossary of notation at the back.

The idea of using conjugation-invariant functions on $\mathbf{G}$ to construct a family of commuting functions, the proof of the existence of loop-regular elements in the Toda phase space and other results in Sections 3 and 4 were first presented in Quinn's thesis [Qu]. The authors wish to thank Victor Guillemin, David Vogan and Allen Knutson for helpful conversations. Singer gratefully acknowledges the support of the Bunting Institute of Radcliffe College and ONR grant \#N00014-89-J-3112.

\section{Background.}

In this section we fix some notation and review the definition of the Toda phase space. The most difficult technical point is the construction (due to Goodman and Wallach $[\mathbf{G W}]$ ) of an extended loop algebra that has an associated group, as it is not true that every infinite-dimensional Lie algebra has an associated Lie group.

First we recall a few standard facts from Lie theory. See for example $[\mathbf{H}]$ or $[\mathbf{K n}]$ for the finite dimensional theory; for facts concerning infinitedimensional Lie algebras and groups see $[\mathbf{K}]$ and $[\mathbf{P S}]$. Take $\mathfrak{g}$ to be a simple finite-dimensional Lie algebra over $\mathbb{C}$ of rank $\ell$, with corresponding connected and simply-connected Lie group $\mathbf{G}$. Let $\mathrm{B}(\cdot, \cdot)$ denote the Killing form on $\mathfrak{g}$. Fix a Cartan subalgebra $\mathfrak{h} \subset \mathfrak{g}$, with corresponding Cartan subgroup $\mathbf{H}$, and let $\Delta$ denote the set of roots associated to the pair $(\mathfrak{g}, \mathfrak{h})$.

Pick a set $\Pi=\left\{\alpha_{1}, \ldots, \alpha_{\ell}\right\}$ of simple roots and let $\Delta_{+}$denote the corresponding positive roots. Let $\alpha_{*}$ denote the highest root so that $\alpha_{*}=\sum k_{i} \alpha_{i}$ for some positive integers $k_{i}$. Choose a Chevalley basis $\left\{E_{\alpha}\right\}_{\alpha \in \Delta} \cup\left\{H_{\alpha_{i}}\right\}_{\alpha_{i} \in \Pi}$ for $\mathfrak{g}$, so that $E_{\alpha}$ belongs to the $\alpha$ rootspace, and $H_{\alpha}=\left[E_{\alpha}, E_{-\alpha}\right]$ is nor- 
malised by $\alpha\left(H_{\alpha}\right)=2$. Then the real span of

$$
\left\{i H_{\alpha}, E_{\alpha}-E_{-\alpha}, i\left(E_{\alpha}+E_{-\alpha}\right): \alpha \in \Delta\right\}
$$

gives a compact real form $\mathfrak{k}$ of $\mathfrak{g}$. Let $\mathbf{K}$ be the corresponding compact real subgroup of $\mathbf{G}$. Then one has Cartan decompositions $\mathfrak{g}=\mathfrak{k}+i \mathfrak{k}$ and $\mathbf{G}=\mathbf{K} \exp (i \mathfrak{k})$. Let $\mathfrak{a}$ be the real span of $\left\{H_{\alpha}\right\}_{\alpha \in \Delta}$, and $\mathfrak{n}$ the complex span of $\left\{E_{\alpha}\right\}_{\alpha \in \Delta_{+}}$. Take $\mathbf{A}$ and $\mathbf{N}$ to be the corresponding subgroups of $\mathbf{G}$. Then one has Iwasawa decompositions $\mathfrak{g}=\mathfrak{k}+\mathfrak{a}+\mathfrak{n}$ and $\mathbf{G}=\mathbf{K A N}$. We follow convention in letting $\mathbf{B}$ denote the group $\mathbf{A N}$ and letting $\mathfrak{b}$ denote its Lie algebra.

As an example consider $\operatorname{sl}(n, \mathbb{C})=s u(n)+\mathfrak{a}_{n}+\mathfrak{n}_{n}$, where $\mathfrak{a}_{n}$ denotes the algebra of real diagonal traceless $n \times n$ matrices, and $\mathfrak{n}_{n}$ is the algebra of strictly upper-triangular $n \times n$ matrices. The corresponding group factorization is $S L(n, \mathbb{C})=S U(n) \mathbf{A}_{n} \mathbf{N}_{n}$, where $\mathbf{A}_{n}$ is the group of diagonal $n \times n$ matrices of determinant one with real positive entries, and $\mathbf{N}_{n}$ is the unipotent group of upper-triangular matrices with 1's on the diagonal. It follows that $\mathbf{B}_{n}$ is the group of upper triangular matrices of determinant 1 with real, positive diagonal entries.

In general $\mathbf{G}$ can be realized as a linear subgroup of $S L(n, \mathbb{C})$ for some $n$, in such a way that if $g \in \mathbf{G}$ has $g=k(g) a(g) n(g)$ as its Iwasawa decomposition in $S L(n, \mathbb{C})$ then each factor belongs to $\mathbf{G}$ and this factorization corresponds to the Iwasawa decomposition in $\mathbf{G}$. (See [H, p. 257] for a discussion of Iwasawa decompositions.) We fix such a linear embedding of $\mathbf{G}$, and identify $\mathfrak{g}$ with the corresponding Lie subalgebra of $\operatorname{sl}(n, \mathbb{C})$.

We describe now some specific infinite-dimensional groups and algebras for which there are decompositions directly analogous to the ones described above (the construction is taken from $[\mathbf{G W}]$ ). Suppose that $w$ is a symmetric weight function on $\mathbb{Z}$, i.e., $w$ is a positive function on the integers such that $w(k+m) \leq w(k) w(m)$, and $w(k)=w(-k)$. In addition suppose that $w$ is of non-analytic type: $\lim _{k \rightarrow \infty} w(k)^{1 / k}=1$, and is rapidly increasing at infinity: $\lim _{k \rightarrow \infty}|k|^{-1 / \lambda} \log w(k)=\infty$ for some $\lambda \in(1,2)$. (For example take $w(k)=\exp \left(|k|^{\frac{2}{3}}\right)$.) Take $A_{w}$ to be the space of functions $f(z)=\sum_{k} a_{k} z^{k}$ on $S^{1}$ satisfying

$$
\|f\|_{w}:=\sum_{k \in \mathbb{Z}}\left|a_{k}\right| w(k)<\infty,
$$

and let $M_{n}\left(A_{w}\right)$ denote the Banach algebra of $n \times n$ matrices with entries in $A_{w}$, with norm given by $\|T\|_{w}:=\left\{\sum_{i, j}\left\|T_{i, j}\right\|_{w}^{2}\right\}^{\frac{1}{2}}$. Define

$$
\begin{aligned}
& S L_{n}\left(A_{w}\right)=\left\{g \in M_{n}\left(A_{w}\right): \operatorname{det}(g(z))=1\right\}, \\
& S U_{n}\left(A_{w}\right)=\left\{g \in S L_{n}\left(A_{w}\right): g(z) \in S U(n) \forall z \in S^{1}\right\},
\end{aligned}
$$




$$
\mathbf{N}_{n}\left(A_{w}\right)=\left\{g \in S L_{n}\left(A_{w}\right): g(z)=\sum_{k \geq 0} a_{k} z^{k}, a_{0} \in \mathbf{N}\right\} .
$$

Each of these sets is a Banach-Lie group, with corresponding Banach-Lie algebra

$$
\begin{aligned}
\operatorname{sl}_{n}\left(A_{w}\right) & =\left\{x \in M_{n}\left(A_{w}\right): \operatorname{tr}(x)=0\right\}, \\
s u_{n}\left(A_{w}\right) & =\left\{x \in \operatorname{sl}_{n}\left(A_{w}\right): x(z) \in \operatorname{su}(n) \forall z \in S^{1}\right\}, \\
\mathfrak{n}_{n}\left(A_{w}\right) & =\left\{x \in \operatorname{sl}_{n}\left(A_{w}\right): x(z)=\sum_{k \geq 0} a_{k} z^{k}, a_{0} \in \mathfrak{n}\right\} .
\end{aligned}
$$

One has Cartan and Iwasawa decompositions analogous to the finite-dimensional case:

$$
\begin{aligned}
s l_{n}\left(A_{w}\right) & =s u_{n}\left(A_{w}\right)+i s u_{n}\left(A_{w}\right)=s u_{n}\left(A_{w}\right)+\mathfrak{a}_{n}+\mathfrak{n}_{n}\left(A_{w}\right) \\
S L_{n}\left(A_{w}\right) & =S U_{n}\left(A_{w}\right) \exp \left(i s u_{n}\left(A_{w}\right)\right)=S U_{n}\left(A_{w}\right) \mathbf{A}_{n} \mathbf{N}_{n}\left(A_{w}\right) .
\end{aligned}
$$

Now define

$$
\begin{aligned}
\mathrm{L} \mathfrak{g} & =\left\{x \in \operatorname{sl}_{n}\left(A_{w}\right): x(z) \in \mathfrak{g} \forall z \in S^{1}\right\}, \\
\mathbf{L G} & =\left\{g \in S L_{n}\left(A_{w}\right): g(z) \in \mathbf{G} \forall z \in S^{1}\right\} .
\end{aligned}
$$

LG is a complex Lie subgroup of $S L_{n}\left(A_{w}\right)$ with Banach-Lie algebra Lg. The algebra and group decompose as:

$$
\begin{aligned}
\mathrm{L} \mathfrak{g} & =\tilde{\mathfrak{k}}+i \tilde{\mathfrak{k}}=\tilde{\mathfrak{k}}+\mathfrak{a}+\tilde{\mathfrak{n}} \\
\mathrm{L} \mathbf{G} & =\widetilde{\mathbf{K}} \exp (i \tilde{\mathfrak{k}})=\widetilde{\mathbf{K}} \mathbf{A} \tilde{\mathbf{N}}
\end{aligned}
$$

where $\tilde{\mathfrak{k}}=\mathrm{L} \mathfrak{g} \cap s u_{n}\left(A_{w}\right), \tilde{\mathfrak{n}}=\mathrm{L} \mathfrak{g} \cap \mathfrak{n}_{n}\left(A_{w}\right), \widetilde{\mathbf{K}}=\mathrm{L} \mathbf{G} \cap S U_{n}\left(A_{w}\right)$ and $\tilde{\mathbf{N}}=$ LG $\cap \mathbf{N}_{n}\left(A_{w}\right)$. Define $\tilde{\mathfrak{b}}=\mathfrak{a}+\tilde{\mathfrak{n}}$ and $\widetilde{\mathbf{B}}=\mathbf{A} \widetilde{\mathbf{N}}$ so that we have

$$
\mathrm{L} \mathfrak{g}=\tilde{\mathfrak{k}}+\tilde{\mathfrak{b}}, \quad \mathrm{L} \mathbf{G}=\widetilde{\mathbf{K}} \widetilde{\mathbf{B}} .
$$

Note that elements of $\widetilde{\mathbf{B}}$ are analytic functions on the open unit disk whose power series converges absolutely to a smooth function on the unit circle.

From the Killing form $\mathrm{B}(\cdot, \cdot)$ on $\mathfrak{g}$ one obtains a non-degenerate bilinear form on $\mathrm{L} \mathfrak{g}$ by integration over $S^{1}$, which by an abuse of notation we also denote $\mathrm{B}(\cdot, \cdot)$ :

$$
\mathrm{B}(\phi, \psi)=\frac{1}{2 \pi} \int_{0}^{2 \pi} \mathrm{B}\left(\phi\left(e^{i \theta}\right), \psi\left(e^{i \theta}\right)\right) d \theta
$$


Let $D=z \frac{d}{d z}$. This derivation gives a natural 2-cocycle $\omega$ on $\mathrm{Lg}$ defined by $\omega(\phi, \psi)=\mathrm{B}(D \phi, \psi)$. This $\mathbb{C}$-valued skew-symmetric bilinear form can be used to construct a central extension of $\mathrm{Lg}$, which we denote $\widetilde{\mathrm{Lg}}$.

Goodman and Wallach [GW] have shown that, provided the weight function $w$ is of non-analytic type and is rapidly increasing at infinity, there exists a Banach-Lie group whose Lie algebra is $\widetilde{\mathrm{Lg}}$. This group is a central extension of LG by $\mathbb{C}^{\times}$, and we denote it $\widetilde{\mathrm{LG}}$. One also has Cartan and Iwasawa decompositions:

$$
\begin{aligned}
\widetilde{\mathrm{L} g} & =\hat{\mathfrak{k}}+i \hat{\mathfrak{k}}=\hat{\mathfrak{k}}+\hat{\mathfrak{a}}+\hat{\mathfrak{n}} \\
\widetilde{\mathrm{LG}} & =\hat{\mathbf{K}} \exp (i \hat{\mathfrak{k}})=\hat{\mathbf{K}} \hat{\mathbf{A}} \hat{\mathbf{N}} .
\end{aligned}
$$

$\hat{\mathbf{K}}$ is a central extension of $\widetilde{\mathbf{K}}$ by $\mathrm{S}^{1}, \hat{\mathbf{A}}=\mathbf{A} \mathbb{R}^{+}$, and $\hat{\mathbf{N}} \cong \widetilde{\mathbf{N}}$, (correspondingly $\hat{\mathfrak{k}}=\tilde{\mathfrak{k}}+i \mathbb{R}, \hat{\mathfrak{a}}=\mathfrak{a}+\mathbb{R}$, and $\hat{\mathfrak{n}} \cong \tilde{\mathfrak{n}})$.

Set $\hat{\mathfrak{b}}=\hat{\mathfrak{a}}+\hat{\mathfrak{n}}$ and $\hat{\mathbf{B}}=\hat{\mathbf{A}} \hat{\mathbf{N}}$. We shall apply the Kostant-Symes approach to the splitting $\widetilde{\mathrm{Lg}}=\hat{\mathfrak{k}}+\hat{\mathfrak{b}}$, and construct the Toda phase space as a coadjoint orbit of $\hat{\mathbf{B}}$.

The next step is to calculate the coadjoint action of $\widetilde{\mathrm{LG}}$ on $\widetilde{\mathrm{Lg}}$; we follow the treatment in $[\mathbf{P S}]$. Because $\widetilde{\mathrm{LG}}$ is a central extension its coadjoint action comes from an action of LG. The adjoint action of $\mathrm{Lg}$ on $\widetilde{\mathrm{Lg}}$ is

$$
\widetilde{\operatorname{ad}}_{\phi}(\psi, q)=\left(\operatorname{ad}_{\phi} \psi, \omega(\phi, \psi)\right),
$$

and this in turn comes from an adjoint action of the group LG on $\widetilde{\mathrm{Lg}}$. If $\gamma \in \mathbf{L} \mathbf{G}$ and $(\psi, q) \in \widetilde{\mathrm{Lg}}$ one has

$$
\widetilde{\operatorname{Ad}}_{\gamma}(\psi, q)=\left(\operatorname{Ad}_{\gamma} \psi, q+\mathrm{B}\left(\gamma^{-1} D \gamma, \psi\right)\right) .
$$

From now on we shall treat $\widetilde{\mathrm{Lg}}=\mathrm{Lg}+\mathbb{C}$ as an algebra over $\mathbb{R}$. We introduce a nondegenerate real-bilinear pairing $\langle\cdot, \cdot\rangle$ on $\widetilde{\mathrm{Lg}}$ defined by

$$
\langle(\phi, p),(\psi, q)\rangle=\operatorname{Re}\{\mathrm{B}(\phi, \psi)+p q\} .
$$

Using this form one can embed $\widetilde{\mathrm{Lg}} \subset(\widetilde{\mathrm{Lg}})^{*}$ as a real subspace, and the image of this embedding is invariant under the co-adjoint action of LG. In fact for $\gamma \in \mathbf{L G}$ and $(\phi, p) \in \widetilde{\mathrm{Lg}} \subset(\widetilde{\mathrm{Lg}})^{*}$, the co-adjoint action of $\gamma$ on $(\phi, p)$ is given by

$$
\widetilde{\operatorname{Ad}}_{\gamma}^{*}(\phi, p)=\left(\operatorname{Ad}_{\gamma} \phi+p \gamma D \gamma^{-1}, p\right) .
$$

This action is sometimes called the gauge action of $\gamma \in \mathbf{L G}$ on $(\phi, p) \in \widetilde{\mathrm{Lg}}$. If two elements of $\widetilde{\mathrm{Lg}}$ are conjugate under this action we shall refer to them as being gauge-conjugate. 
Finally, we are in a position to construct the (symmetric, real, periodic, tridiagonal) Toda phase space as a coadjoint orbit of the group $\hat{\mathbf{B}}$. From the splitting $\widetilde{\mathrm{Lg}}=\hat{\mathfrak{k}}+\hat{\mathfrak{b}}$ it follows that $\widetilde{\mathrm{Lg}}{ }^{*}=\hat{\mathfrak{k}}^{*}+\hat{\mathfrak{b}}^{*}$. Using the form $\langle\cdot, \cdot\rangle$ one has $\hat{\mathfrak{k}}^{\perp} \subset \operatorname{Ann}(\hat{\mathfrak{k}}) \cong \hat{\mathfrak{b}}^{*}$, and this subspace is invariant under the co-adjoint action of $\hat{\mathbf{B}}$ for if $\gamma \in \hat{\mathbf{B}}$ and $(\phi, p) \in \hat{\mathfrak{k}}^{\perp}$ the co-adjoint action of $\gamma$ on $(\phi, p)$ is given by

$$
\Pi_{\hat{\mathfrak{k}} \perp} \widetilde{\operatorname{Ad}}_{\gamma}^{*}(\phi, p) .
$$

Here $\Pi_{\hat{\mathfrak{k}} \perp}$ denotes projection onto $\hat{\mathfrak{k}}^{\perp}$ along $\hat{\mathfrak{b}}^{\perp}$. One can easily verify that $\widetilde{\mathrm{Lg}}=\hat{\mathfrak{k}}^{\perp}+\hat{\mathfrak{b}}^{\perp}$ where $\hat{\mathfrak{k}}^{\perp}=i \hat{\mathfrak{k}}$ and $\hat{\mathfrak{b}}^{\perp}=i \hat{\mathfrak{b}}$.

The symmetric periodic Toda phase space is defined to be the orbit of $\hat{\mathbf{B}}$ through the element $\left(\phi_{o}, 1\right) \in \hat{\mathfrak{k}}^{\perp}$ where

$$
\phi_{o}(z)=\sum_{i}\left(E_{\alpha_{i}}+E_{-\alpha_{i}}\right)+E_{\alpha_{*}} z^{-1}+E_{-\alpha_{*}} z,
$$

(recall that $\alpha_{*}=\sum k_{i} \alpha_{i}$ is the highest root of $\mathfrak{g}$ ). This orbit consists of all elements of the form $(\phi, 1)$ with

$$
\phi=H+\sum_{i} q_{i}\left(E_{\alpha_{i}}+E_{-\alpha_{i}}\right)+q_{*}\left(E_{\alpha_{*}} z^{-1}+E_{-\alpha_{*}} z\right),
$$

where $q_{i}, q_{*}>0$ satisfy $q_{*} \cdot \prod q_{i}^{k_{i}}=1$, and $H$ belongs to $\mathfrak{a}$. The periodic Toda Hamiltonian is $\mathrm{H}(\phi)=\frac{1}{2} \mathrm{~B}(\phi, \phi)$.

We shall apply the involution theorem of Kostant and Symes and the factorization theorem of Adler and van Moerbeke and Reyman and SemenovTian-Shansky in this infinite dimensional setting. For the details of these theorems see $[\mathbf{R S}]$. Because $\widetilde{\mathrm{Lg}}=\hat{\mathfrak{k}}+\hat{\mathfrak{b}}$ is a splitting of $\widetilde{\mathrm{Lg}}$ as a vector sum of subalgebras and because $(0,1) \in \widetilde{\mathrm{Lg}}^{*}$ satisfies $\langle(0,1),[\hat{\mathfrak{k}}, \hat{\mathfrak{k}}]\rangle=0$ and $\langle(0,1),[\hat{\mathfrak{b}}, \hat{\mathfrak{b}}]\rangle=0$ we can apply the involution theorem to show that the restrictions of any coadjoint-invariant functions on $\widetilde{\mathrm{Lg}}$ to the Toda phase space will commute in the standard Poisson structure. Furthermore, the factorization theorem tells us that if $F$ is a coadjoint-invariant function then the Hamiltonian flow associated to $F$ on the Toda phase space is given by

$$
(\phi(t), 1)=\widetilde{\operatorname{Ad}}_{b(t)}^{*}(\phi(0), 1),
$$

where $\exp t \nabla F(\phi(0), 1)=k(t)^{-1} b(t)$ with $k(t) \in \hat{\mathbf{K}}$ and $b(t) \in \hat{\mathbf{B}}$.

\section{Loop-regularity and invariant functions.}

We shall construct a family of Poisson-commuting functions on the Toda phase space by an application of the Kostant-Symes involution theorem. 
To do so we require invariant functions on $(\widetilde{\mathrm{Lg}})^{*}$ (or, more precisely, on $\left.\widetilde{\mathrm{Lg}} \subset(\widetilde{\mathrm{Lg}})^{*}\right)$. Roughly speaking, coadjoint orbits in $\widetilde{\mathrm{Lg}} \subset(\widetilde{\mathrm{Lg}})^{*}$ correspond to conjugacy classes in $\mathbf{G}$, and so a natural way to get invariant functions on $\widetilde{\mathrm{Lg}}$ is via the class functions of $\mathbf{G}$. We make this precise as follows.

Definition 3.1. Given $(\phi, p) \in \widetilde{\mathrm{Lg}}$ such that $p \neq 0$ the differential equation

$$
\dot{f}(t) f(t)^{-1}=\frac{1}{i p} \phi\left(e^{i t}\right), \quad f(0)=\mathrm{Id},
$$

where $f: \mathbb{R} \rightarrow \mathbf{G}$ is called the monodromy equation associated to $(\phi, p)$. The monodromy $\mathrm{M}_{(\phi, p)}$ of the element $(\phi, p) \in \widetilde{\mathrm{Lg}}$ is defined by $\mathrm{M}_{(\phi, p)}=f(2 \pi)$, and we call

$$
\mathrm{M}: \widetilde{\mathrm{Lg}} \rightarrow \mathbf{G}:(\phi, p) \mapsto \mathrm{M}_{(\phi, p)}
$$

the monodromy map. An element $(\phi, p) \in \widetilde{\mathrm{Lg}}$ will be said to be loop-regular if its monodromy is regular, i.e. if the normalizer of $\mathbf{M}_{(\phi, p)}$ in $\mathbf{G}$ is conjugate to the Cartan subgroup $\mathbf{H}$.

The monodromy equation can be thought of as describing the flow of a time- and parameter-dependent vector field on a space of matrices. (Recall that we have fixed a matrix representation of $\mathfrak{g}$ and G.) Since the parameter space, namely $\widetilde{\mathrm{Lg}}$, is a Banach space it follows, using standard results from the theory of differential equations, that the flow depends smoothly on $\widetilde{\mathrm{Lg}}$ and that the monodromy map is smooth. See for example $[\mathbf{L}]$. Thus the loop-regular elements form an open subset of $\widetilde{\mathrm{Lg}}$, and in Section 4 we show that they are dense in the Toda phase space.

As an aside we also note that for fixed $(\phi, p)$ a solution $f(t)$ to the monodromy equation exists for all $t \in \mathbb{R}$. To see this consider the trivial principal bundle $\mathbf{G} \hookrightarrow S^{1} \times \mathbf{G} \rightarrow S^{1}$ (whose space of connections can be identified with $\left.\Omega^{1}\left(\mathrm{~S}^{1}\right) \otimes \mathfrak{g} \cong C^{\infty}\left(\mathrm{S}^{1}, \mathfrak{g}\right)\right)$. The horizontal lift of the curve $c(t): t \mapsto \exp (i t)$ through $(1, \mathrm{Id})$ determined by the connection $\frac{1}{i p} \phi$ is given by $(c(t), f(t))$ where $f(t)$ solves the monodromy equation for $(\phi, p)$. From the theory of principal bundles if follows that the solution $f(t)$ exists for all $t \in \mathbb{R}$.

The following proposition from $[\mathrm{PS}]$ gives a parametrization of the gauge co-adjoint orbits and allows one to describe the invariant functions on $\widetilde{\mathrm{Lg}} \subset$ $(\widetilde{\mathrm{Lg}})^{*}$.

Proposition 3.1 [PS]. The monodromy classifies the gauge co-adjoint orbits in the following sense:

(1) If $\gamma \in \mathbf{L G}$, then the monodromy of $\widetilde{\operatorname{Ad}}_{\gamma}^{*}(\phi, p)$ is $\gamma(1) \mathrm{M}_{(\phi, p)} \gamma(1)^{-1}$. 
(2) If $\mathrm{M}_{(\phi, p)}$ and $\mathrm{M}_{(\tilde{\phi}, p)}$ are conjugate in $\mathbf{G}$, then $(\phi, p)$ and $(\tilde{\phi}, p)$ are gauge-conjugate in $\widetilde{\mathrm{Lg}}$. Specifically, if $\mathrm{M}_{(\phi, p)}=g \mathrm{M}_{(\tilde{\phi}, p)} g^{-1}$ for some $g \in \mathbf{G}$, and if $f$ and $\tilde{f}$ solve the monodromy equation for $(\phi, p)$ and $(\tilde{\phi}, p)$ respectively, then $(\phi, p)=\widetilde{\operatorname{Ad}}_{\gamma}^{*}(\tilde{\phi}, p)$ where $\gamma\left(e^{i t}\right)=f(t) g \tilde{f}(t)^{-1}$. Hence for fixed $p \neq 0$, the monodromy map gives a 1-1 correspondence between coadjoint orbits of $\mathbf{L G}$ in $L \mathfrak{g} \times\{p\} \in \widetilde{\mathrm{Lg}}$ and conjugacy classes of G.

A simple corollary to this proposition is that loop-regular elements can be conjugated to $\mathfrak{h} \times \mathbb{C}^{\times}$under the gauge action:

Corollary 3.1. For any loop-regular $(\phi, p)$ there exists $\gamma \in \mathbf{L G}$ and a constant loop $\mu \in \mathfrak{h} \subset \mathrm{Lg}$ such that $(\phi, p)=\widetilde{\operatorname{Ad}}_{\gamma}^{*}(\mu, p)$.

Proof. By loop-regularity $\mathrm{M}_{(\phi, p)}$ is conjugate to some $x \in \mathbf{H}$. Choose $\xi \in \mathfrak{h}$ such that $x=\exp \xi$ and set $\mu=\frac{i p}{2 \pi} \xi$. By construction $\mathrm{M}_{(\mu, p)}=x$, so that $\mathrm{M}_{(\phi, p)}$ and $\mathrm{M}_{(\mu, p)}$ are conjugate in $\mathbf{G}$. Hence by the second part of Proposition 3.1, $(\phi, p)$ and $(\mu, p)$ are gauge-conjugate. I.e. there exists $\gamma \in$ LG with $(\phi, p)=\widetilde{\operatorname{Ad}}_{\gamma}^{*}(\mu, p)$.

In the case of loop-regular elements in the Toda phase space the same argument can be strengthened to the following:

Corollary $3.2 \quad[\mathbf{Q u}] . \quad$ For any loop-regular $(\phi, 1)$ in the Toda phase space there exists $\gamma \in \widetilde{\mathbf{K}} \subset \widetilde{\mathrm{LG}}$, and $\mu \in \mathfrak{a} \subset \mathfrak{h}$, such that $(\phi, 1)=\widetilde{\operatorname{Ad}}_{\gamma}^{*}(\mu, 1)$.

Proof. The monodromy equation for $(\phi, 1)$ is $\dot{f}(t) f^{-1}(t)=\frac{1}{i} \phi\left(e^{i t}\right)$. Since $\phi(z) \in i \tilde{\mathfrak{k}}$, this shows that $f(t) \in \mathbf{K}$ for all $t$, and in particular the monodromy $f(2 \pi)=\mathrm{M}_{(\phi, 1)}$ is in $\mathbf{K}$. By loop-regularity, and since $\mathbf{K}$ is the compact form of $\mathbf{G}, \mathbf{M}_{(\phi, 1)}$ can be conjugated in $\mathbf{K}$ to an element of the maximal torus $\mathbf{H} \cap \mathbf{K}$ of $\mathbf{K}$. Thus there exists $k \in \mathbf{K}$ and $x \in \mathbf{H} \cap \mathbf{K}$ with $\mathrm{M}_{(\phi, 1)}=k x k^{-1}$.

Choose $\xi \in \mathfrak{h} \cap \mathfrak{k}=i \mathfrak{a}$ such that $x=\exp \xi$ and set $\mu=\frac{i}{2 \pi} \xi$. By construction $\tilde{f}(t)=\exp \frac{t}{2 \pi} \xi$ solves the monodromy equation for $(\mu, 1)$, and $\mathrm{M}_{(\mu, 1)}=$ $x$ is conjugate to $\mathrm{M}_{(\phi, 1)}$. Hence $(\phi, 1)$ and $(\mu, 1)$ are gauge-conjugate by Proposition 3.1, and specifically one has $(\phi, 1)=\widetilde{\operatorname{Ad}}_{\gamma}^{*}(\mu, 1)$ where $\gamma\left(e^{i t}\right)=$ $f(t) k \tilde{f}(t)^{-1}$. By construction $\mu \in \mathfrak{a}$, and the loop $\gamma$ has image in $\mathbf{K}$, so $\gamma \in \widetilde{\mathbf{K}}$ as required.

We now define a family of invariant functions on $\widetilde{\mathrm{Lg}}$ which, when restricted to the Toda phase space, will give rise to a completely integrable system. We 
must first define a factorization. Given $g \in \mathbf{G}$ let $\mathrm{k}[g]$ denote the $\mathbf{K}$ factor of $g$ in the Cartan decomposition $\mathbf{G}=\mathbf{K} \exp (i \mathfrak{k})$. Next, let $\Psi_{1}, \ldots, \Psi_{\ell}$ denote the characters of the irreducible representations corresponding to the fundamental weights of $\mathbf{G}$. Then $d \Psi_{1}, \ldots, d \Psi_{\ell}$ are independent at regular values of $\mathbf{G}$ [St, p. 123], and because the fundamental weights occur in dual pairs the characters occur in conjugate pairs. Thus their real and imaginary parts give a collection of $\ell$ real-valued class functions on $\mathbf{G}$ which are also functionally independent at regular values of $\mathbf{G}$. We denote this collection $\chi_{1}, \ldots, \chi_{\ell}$.

Definition 3.2. For $j=1, \ldots, \ell$, define

$$
F_{j}: \widetilde{\mathrm{Lg}} \rightarrow \mathbb{R}:(\phi, p) \mapsto \chi_{j}\left(\mathrm{k}\left[\mathrm{M}_{(\mu, p)}\right]\right)
$$

where $(\mu, p)$ is any loop in $\mathfrak{h} \times \mathbb{C}$ gauge-conjugate to $(\phi, p)$.

For $\mu \in \mathfrak{h}$ one has $\mathrm{M}_{(\mu, p)}=\exp \left(\frac{2 \pi}{i} \frac{\mu}{p}\right)$, and $\mathrm{k}\left[\mathrm{M}_{(\mu, p)}\right]=\exp \left(\frac{2 \pi}{i} \Pi_{\mathfrak{a}} \frac{\mu}{p}\right)$, so that an equivalent expression for $F_{j}$ is

$$
F_{j}(\phi, p)=\chi_{j}\left(\exp \left(\frac{2 \pi}{i} \Pi_{\mathfrak{a}} \frac{\mu}{p}\right)\right)
$$

whenever $(\phi, p)$ is gauge-conjugate to $(\mu, p)$. To see that $F_{j}$ is well-defined suppose that $(\mu, p)$ and $(\tilde{\mu}, p)$ are gauge-conjugate elements of $\mathfrak{h} \times \mathbb{C}$. Then they are conjugate by an element of the affine Weyl group so $\tilde{\mu}=w \cdot \mu+p \lambda$ for some $w$ in the ordinary Weyl group, and $\lambda$ in the coroot lattice. Since $\exp (2 \pi i \lambda)=$ Id and the Weyl group preserves $\mathfrak{a}$ it follows that

$$
\exp \left(\frac{2 \pi}{i} \Pi_{\mathfrak{a}} \frac{\tilde{\mu}}{p}\right)=\exp \left(w \cdot \frac{2 \pi}{i} \Pi_{\mathfrak{a}} \frac{\mu}{p}\right)=k \exp \left(\frac{2 \pi}{i} \Pi_{\mathfrak{a}} \frac{\mu}{p}\right) k^{-1}
$$

for some $k \in \mathbf{K}$. Hence the functions $F_{j}$ are well-defined and invariant under the gauge action.

In order to analyze the flows induced by the functions $F_{j}$ on the Toda phase space we introduce a family of locally defined functions that are simpler to work with. To construct these local functions we first show that gauge-conjugation to $\mathfrak{h} \times \mathbb{C}^{\times}$is (locally) a well defined map.

Proposition 3.2. Fix any loop-regular $(\psi, q)$ in $\widetilde{\mathrm{Lg}}$ and any $(\xi, q) \in$ $\mathfrak{h} \times \mathbb{C}^{\times}$gauge-conjugate to $(\psi, q)$. There exists a gauge-invariant loop-regular neighborhood $U \subset \widetilde{\mathrm{Lg}}$ of $(\psi, q)$, and a smooth gauge-invariant map

$$
\eta: U \rightarrow \mathfrak{h} \times \mathbb{C}^{\times}
$$

such that $\eta(\psi, q)=(\xi, q)$, and $\eta(\phi, p)$ is gauge-conjugate to $(\phi, p)$ for each $(\phi, p) \in U$. 
Proof. This proposition follows from the implicit function theorem for Banach manifolds. (See, for example, $[\mathbf{A M R}]$ for properties of Banach manifolds.) We sketch the argument.

Let $\Psi=\left(\Psi_{1}, \ldots, \Psi_{\ell}\right)$ denote the vector of character maps corresponding to the fundamental weights of $\mathfrak{g}$. Define $F: \widetilde{\mathrm{Lg}} \times\left(\mathfrak{h} \times \mathbb{C}^{\times}\right) \rightarrow \mathbb{C}^{l} \times \mathbb{C}$ by

$$
((\phi, p),(\mu, r)) \mapsto\left(\Psi\left(\mathrm{M}_{(\phi, p)}\right)-\Psi\left(\mathrm{M}_{(\mu, r)}\right), p-r\right) .
$$

Then by hypothesis $F((\psi, q),(\xi, q))=(0,0)$, and one can easily check that the partial derivative of $F((\psi, q),(\xi, q))$ with respect to $(\xi, q)$ is invertible, (the loop-regularity of $(\xi, q)$ is needed here). So by the implicit function theorem there is a neighborhood $\tilde{U}$ of $(\psi, q)$ and a smooth map $\tilde{\eta}: \tilde{U} \mapsto \mathfrak{h} \times \mathbb{C}^{\times}$ such that $F((\phi, p), \tilde{\eta}(\phi, p))=(0,0)$, and $\tilde{\eta}(\psi, q)=(\xi, q)$. Since $(\xi, q)$ is loop-regular its stabiliser in the affine Weyl group is trivial, and there is a neighborhood of $(\xi, q)$ in $\mathfrak{h} \times \mathbb{C}^{\times}$on which no two elements are gaugeconjugate. Shrinking $\tilde{U}$ if necessary we can assume that $\tilde{\eta}(\tilde{U})$ lies in such a neighborhood. Now set $U=\widetilde{\operatorname{Ad}}_{\mathrm{LG}}^{*} \tilde{U}$ and define $\eta: U \rightarrow \mathfrak{h} \times \mathbb{C}^{\times}$to be the map which first gauge-conjugates $(\phi, p) \in U$ to $\tilde{U}$, then maps to $\mathfrak{h} \times \mathbb{C}^{\times}$by $\tilde{\eta}$. By construction $\eta$ has the desired properties. (Note that since the set of loop-regular elements is open in $\widetilde{\mathrm{Lg}}$, we can without loss of generality take all the open sets constructed to be loop-regular.)

Quinn has proved a stronger version of this result in his thesis: The map $\eta$ can be extended smoothly to the central extension of the algebra of smooth loops in $\mathfrak{g}[\mathbf{Q u}]$. In that case the theory of Banach manifolds does not apply, and one needs the Nash-Moser implicit function theorem.

Let $\hat{\eta}$ denote the composition of projection onto $\mathfrak{h}$ with $\eta$. (For example, in the proposition above $\hat{\eta}(\psi, q)=\xi$.) We now define a family of gaugeinvariant functions on the set $U$ constructed in Proposition 3.2.

Definition 3.3. For $j=1, \ldots, \ell$, define

$$
I_{j}: U \rightarrow \mathbb{R}:(\phi, p) \mapsto \operatorname{Re}\left[\alpha_{j}\left(\frac{\hat{\eta}(\phi, p)}{p}\right)\right]=\alpha_{j}\left(\Pi_{\mathfrak{a}} \frac{\hat{\eta}(\phi, p)}{p}\right) .
$$

Take $\tau^{j} \in \mathfrak{a}, j=1, \ldots, \ell$, to be the dual basis to the simple roots, so that $\alpha_{i}\left(\tau^{j}\right)=\delta_{i j}$. By construction $\sum_{k} \tau^{k} I_{k}(\phi, p)=\Pi_{\mathfrak{a}} \frac{\hat{\eta}(\phi, p)}{p}$, and so

$$
\left.F_{j}\right|_{U}=\chi_{j}\left(\exp \left(\frac{2 \pi}{i} \sum_{k} \tau^{k} I_{k}\right)\right) .
$$

While this characterization of the $F_{j}$ 's holds only locally, we use it to show functional independence of the family. 
We finish this section with a calculation of the gradients of the functions $I_{j}$. The following lemma makes the calculation relatively easy.

Lemma 3.1. Suppose $(\mu, p)$ is loop-regular and $\mu \in \mathfrak{h}$. Then $\widetilde{\mathrm{Lg}}$ has the vector space splitting

$$
\widetilde{\mathrm{Lg}}=[\mathfrak{h} \times \mathbb{C}] \oplus \widetilde{\operatorname{ad}}_{\mathrm{Lg}}^{*}(\mu, p),
$$

and this splitting is orthogonal with respect to the form $\langle\cdot, \cdot\rangle$.

Proof. To show that $\mathfrak{h} \times \mathbb{C}$ and $\widetilde{\operatorname{ad}}_{\mathrm{Lg}}^{*}(\mu, p)$ are orthogonal is straightforward. Take any $\nu \in \mathrm{Lg}$ and any $\left(\mu^{\prime}, p^{\prime}\right) \in \mathfrak{h} \times \mathbb{C}$. Then

$$
\begin{aligned}
\left\langle\tilde{\operatorname{ad}}_{\nu}^{*}(\mu, p),\left(\mu^{\prime}, p^{\prime}\right)\right\rangle & =\left\langle(\mu, p),-\widetilde{\operatorname{ad}}_{\nu}\left(\mu^{\prime}, p^{\prime}\right)\right\rangle \\
& =-\left\langle(\mu, p),\left(\left[\nu, \mu^{\prime}\right], 0\right)\right\rangle \\
& =-\operatorname{ReB}\left(\mu,\left[\nu, \mu^{\prime}\right]\right) .
\end{aligned}
$$

The last expression is zero by invariance of the Killing form, and because both $\mu$ and $\mu^{\prime}$ lie in the commutative algebra $\mathfrak{h}$.

To show that $\mathfrak{h} \times \mathbb{C}$ and $\widetilde{\operatorname{ad}}_{\mathrm{Lg}}^{*}(\mu, p)$ together span $\widetilde{\mathrm{Lg}}$ it suffices to show that given any $(\psi, q) \in \widetilde{\mathrm{Lg}}$ one can find $\xi \in \mathfrak{h}, r \in \mathbb{C}$, and $\nu \in \mathrm{Lg}$ such that

$$
(\psi, q)=(\xi, r)+\widetilde{\operatorname{ad}}_{\nu}^{*}(\mu, p) .
$$

Suppose $\psi=\sum \psi_{k} z^{k}$, and that $\psi_{k}=\psi_{k}^{o}+\sum_{\alpha \in \Delta} \psi_{k}^{\alpha} E_{\alpha}$ is the rootspace decomposition of $\psi_{k}$ in $\mathfrak{g}$, with $\psi_{k}^{o} \in \mathfrak{h}$. Then $r=q, \xi=\psi_{0}^{o}$, and $\nu=\sum \nu_{k} z^{k}$ with $\nu_{k}=\nu_{k}^{o}+\sum_{\alpha \in \Delta} \nu_{k}^{\alpha} E_{\alpha}$ where

$$
\nu_{k}^{\alpha}=\frac{-1}{p k+\alpha(\mu)} \psi_{k}^{\alpha}, \quad(k \neq 0) \quad \nu_{k}^{o}=\frac{-1}{p k} \psi_{k}^{o} .
$$

(Note that loop-regularity of $(\mu, p)$ ensures that $p k+\alpha(\mu)$ is never zero.) $\nu_{0}^{o} \in \mathfrak{h}$ can be chosen arbitrarily. Thus $[\mathfrak{h} \times \mathbb{C}] \oplus \widetilde{\operatorname{ad}}_{\mathrm{Lg}}^{*}(\mu, p)$ does span $\widetilde{\mathrm{Lg}}$.

That $\mathfrak{h} \times \mathbb{C}$ and $\widetilde{\operatorname{ad}}_{\mathrm{Lg}}^{*}(\mu, p)$ intersect trivially now follows from their orthogonality, and the non-degeneracy of the form $\langle\cdot, \cdot\rangle$. Hence as required $\widetilde{\mathrm{Lg}}=[\mathfrak{h} \times \mathbb{C}] \oplus \widetilde{\operatorname{ad}}_{\mathrm{Lg}}^{*}(\mu, p)$.

For each $\alpha_{j} \in \Pi$ take $h_{j} \in \mathfrak{h}$ to be the unique element satisfying $\alpha_{j}(x)=$ $\mathrm{B}\left(x, h_{j}\right)$ for every $x \in \mathfrak{h}$. (Note in particular that $h_{j} \in \mathfrak{a}$.) The gradient of $I_{j}$ is now straightforward to calculate:

Proposition 3.3. Let $\eta$ and $U$ be as in Proposition 3.2. Suppose $(\phi, p) \in$ $U, \gamma \in \mathbf{L} \mathbf{G}$ and $\mu \in \mathfrak{h}$, are such that $(\phi, p)=\widetilde{\operatorname{Ad}}_{\gamma}^{*}(\mu, p)$ where $\eta(\phi, p)=(\mu, p)$. Then

$$
\nabla I_{j}(\phi, p)=\widetilde{\operatorname{Ad}}_{\gamma}\left(\frac{1}{p} h_{j},-\frac{1}{p^{2}} \alpha_{j}(\mu)\right) .
$$


Proof. Because of the invariance of $I_{j}$ one has $\nabla I_{j}(\phi, p)=\widetilde{\operatorname{Ad}_{\gamma}} \nabla I_{j}(\mu, p)$. Take $(\psi, q) \in \widetilde{\mathrm{Lg}}$ and decompose it as $(\psi, q)=(\xi, q)+\widetilde{\operatorname{ad}}_{\nu}^{*}(\mu, p)$, as in Lemma 3.1, with $\xi \in \mathfrak{h}$ and $\nu \in$ Lg. Then

$$
\left\langle(\psi, q), \nabla I_{j}(\mu, p)\right\rangle=\left\langle(\xi, q), \nabla I_{j}(\mu, p)\right\rangle+\left\langle\widetilde{\operatorname{ad}}_{\nu}^{*}(\mu, p), \nabla I_{j}(\mu, p)\right\rangle .
$$

Note that the second term is zero because of the invariance of $I_{j}$. The first term is $\left.\frac{d}{d t}\right|_{t=0} I_{j}(\mu+t \xi, p+t q)$, and since by construction $\eta$ fixes a neighborhood of $(\mu, p)$ in $\mathfrak{h} \times \mathbb{C}$,

$$
\begin{aligned}
\left\langle(\xi, q), \nabla I_{j}(\mu, p)\right\rangle & =\left.\frac{d}{d t}\right|_{t=0} \operatorname{Re} \alpha_{j}\left(\frac{\mu+t \xi}{p+t q}\right) \\
& =\operatorname{Re}\left(\frac{1}{p} \alpha_{j}(\xi)-q \frac{\alpha_{j}(\mu)}{p^{2}}\right) \\
& =\left\langle(\xi, q),\left(\frac{1}{p} h_{j},-\frac{1}{p^{2}} \alpha_{j}(\mu)\right)\right\rangle .
\end{aligned}
$$

By the orthogonality of $\mathfrak{h} \times \mathbb{C}$ and $\widetilde{\operatorname{ad}}_{\mathrm{Lg}}^{*}(\mu, p)$ the last expression is equal to $\left\langle(\psi, q),\left(\frac{1}{p} h_{j},-\frac{1}{p^{2}} \alpha_{j}(\mu)\right)\right\rangle$, and we get $\nabla I_{j}(\mu, p)=\left(\frac{1}{p} h_{j},-\frac{1}{p^{2}} \alpha_{j}(\mu)\right)$, which completes the proof.

Let $(\phi, 1)$ be a loop-regular element of the Toda phase space. It follows from combining the above proposition with Corollary 3.2 that the $\nabla I_{j}(\phi, 1)$ 's are linearly independent elements of $i \hat{\mathfrak{k}}=i \tilde{\mathfrak{k}}+\mathbb{R}$. Now define $f_{j}: \mathbb{R}^{\ell} \rightarrow$ $\mathbb{R}: \vec{x} \mapsto \chi_{j}\left(\exp \left(\frac{2 \pi}{i} \sum \tau^{k} x_{k}\right)\right)$, so that locally one has $\left.F_{j}\right|_{U}=f_{j}\left(I_{1}, \ldots, I_{\ell}\right)$, and $\nabla F_{j}=\sum_{k} \frac{\partial f_{j}}{\partial x_{k}} \nabla I_{k}$. One can easily check that because of the functional independence of the $\chi_{j}$ 's at regular elements of $\mathbf{G}$ the matrix $\left(\frac{\partial f_{j}}{\partial x_{k}}(\vec{I}(\phi, 1))\right)$ is invertible. Putting these pieces together we arrive at:

Corollary 3.3. Let $F=\sum c_{j} F_{j}$ for some real constants $c_{j}$. If $(\phi, 1)$ is a loop-regular element of the Toda phase space then $\nabla F(\phi, 1) \in i \hat{\mathfrak{k}}$, and furthermore $\nabla F(\phi, 1)=0$ if and only if $c_{1}=\cdots=c_{\ell}=0$.

\section{Existence of loop-regular elements.}

We now show that loop-regular elements form a dense open subset of the Toda phase space.

On $\mathrm{L} \mathfrak{g} \times\{1\}$ the monodromy can be regarded as a map $\mathrm{M}: \mathrm{L} \mathfrak{g} \rightarrow \mathbf{G}: \phi \mapsto$ $f(2 \pi)$, where $f: \mathbb{R} \rightarrow \mathbf{G}$ solves the monodromy equation $\dot{f} f^{-1}=\frac{1}{i} \phi\left(e^{i t}\right)$, 
and $f(0)=$ Id. This map extends to the whole of $C^{\infty}(\mathbb{R}, \mathfrak{g})$, thinking of $\mathrm{L} \mathfrak{g} \subset C^{\infty}(\mathbb{R}, \mathfrak{g})$ as the subspace of $2 \pi$-periodic functions. By an abuse of notation we shall denote this map by the same letter, $\mathrm{M}: C^{\infty}(\mathbb{R}, \mathfrak{g}) \rightarrow \mathbf{G}$, and refer to it as the lift map.

The reason for this nomenclature is that if we consider the trivial principal bundle $\mathbf{G} \hookrightarrow \mathbb{R} \times \mathbf{G} \rightarrow \mathbb{R}$ (whose space of connections can be identified with $\left.\Omega^{1}(\mathbb{R}) \otimes \mathfrak{g} \cong C^{\infty}(\mathbb{R}, \mathfrak{g})\right)$ then the horizontal lift of the curve $c(t): t \mapsto t$ through $(0, \mathrm{Id})$ determined by the connection $\frac{1}{i} \phi$ is given by $(c(t), f(t))$ where $f(t)$ solves the lift equation for $\phi$. This interpretation also shows that the solution $f(t)$ exists for all $t \in \mathbb{R}$.

It is not hard to verify the following lemma.

Lemma 4.1. If $f$ solves the lift equation for $\phi \in C^{\infty}(\mathbb{R}, \mathfrak{g})$ and if $\psi$ is an element of $C^{\infty}(\mathbb{R}, \mathfrak{g})$, then

$$
\mathrm{M}(\phi+\psi)=\mathrm{M}(\phi) \mathrm{M}\left(\operatorname{Ad}_{f^{-1}} \psi\right) .
$$

We can now prove the following proposition:

Proposition 4.1 $[\mathrm{Qu}]$. The set of loop-regular elements is open and dense in the Toda phase space.

Proof. Since the monodromy is a real analytic mapping on the Toda phase space (see for example [CL, p. 36]), it suffices to show that the set of loopregular elements of the Toda phase space is non-empty. We do this by constructing sequences $\phi_{k}, \psi_{k} \in C^{\infty}(\mathbb{R}, \mathfrak{g})$ such that $\phi_{k}+\psi_{k}$ lies in the Toda phase space and the following properties hold:

(1) $\mathrm{M}\left(\phi_{k}\right)=\mathrm{M}_{0}$ is constant in $k$ and $\mathrm{M}_{0}$ is regular in $\mathbf{G}$.

(2) The solution $f_{k}: \mathbb{R} \mapsto \mathbf{G}$ to the lift equation for $\phi_{k}$ has image in the compact subgroup $\mathbf{K} \subset \mathbf{G}$.

(3) $\quad \psi_{k} \in \mathrm{Lg}$ and $\psi_{k} \rightarrow 0$ as $k \rightarrow \infty$.

Properties (2) and (3) force $\operatorname{Ad}_{f_{k}^{-1}} \psi_{k} \rightarrow 0$ as $k \rightarrow \infty$, and so one has $\mathrm{M}\left(\operatorname{Ad}_{f_{k}^{-1}} \psi_{k}\right) \rightarrow$ Id. Thus $\mathrm{M}\left(\phi_{k}+\psi_{k}\right) \rightarrow \mathrm{M}_{0}$ by property (1) and lemma 4.1. Since $\mathrm{M}_{0}$ is regular and the set of regular elements in $\mathbf{G}$ is open, for sufficiently large $k$ the monodromy $\mathrm{M}\left(\phi_{k}+\psi_{k}\right)$ will be regular and hence $\phi_{k}+\psi_{k}$ will be loop-regular. So the problem becomes one of finding a sequence $\phi_{k}+\psi_{k}$ satisfying (1), (2) and (3).

Consider the set $\left\{H_{\alpha}\right\}_{\alpha \in \Delta}$. This forms a root system in $\mathfrak{h}$, with a base given by the simple co-roots $\left\{H_{\alpha_{i}}\right\}_{\alpha_{i} \in \Pi}$. Define $H=\sum_{\alpha>0} H_{\alpha}$, and let $\left\{\tau^{i}: i=1, \ldots, \ell\right\} \subset \mathfrak{h}$ be a dual basis to the set of simple roots so that $\alpha_{i}\left(\tau^{j}\right)=\delta_{i j}$ for all $i, j$. From the theory of root systems one has that

$$
H=\sum_{\alpha>0} H_{\alpha}=\sum_{i=1}^{\ell} d_{i} H_{\alpha_{i}}=2 \sum_{i=1}^{\ell} \tau^{i}
$$


where the coefficients $d_{i}$ belong to $\mathbb{Z}_{>0}$. Define

$$
E=\sum_{i=1}^{\ell} \sqrt{d_{i}} E_{\alpha_{i}}, \quad F=\sum_{i=1}^{\ell} \sqrt{d_{i}} E_{-\alpha_{i}} .
$$

Using the three expressions for $H$ it is easy to verify that the triple $\{H, E, F\}$ generates an algebra isomorphic to $\operatorname{sl}(2, \mathbb{C})$, with $[E, F]=H,[H, E]=2 E$ and $[H, F]=-2 F$. Consequently there exists an element $g \in \mathbf{G}$ such that $E+F=\operatorname{Ad}_{g} H$.

Recalling that $\alpha_{*}=\sum k_{i} \alpha_{i}$ is the highest root of $\mathfrak{g}$ we let $c=\left(\sum k_{i}\right)+1$ and set $p(k)=\frac{1+2 c k}{2 c}$. We can now define the sequences $\phi_{k}, \psi_{k}$ :

$$
\begin{aligned}
& \phi_{k}=p(k)(E+F)=p(k) \sum_{i=1}^{\ell} \sqrt{d_{i}}\left(E_{\alpha_{i}}+E_{-\alpha_{i}}\right), \\
& \psi_{k}=\frac{1}{\prod_{i=1}^{\ell}\left(p(k) \sqrt{d_{i}}\right)^{k_{i}}}\left(E_{\alpha_{*}} z^{-1}+E_{-\alpha_{*}} z\right) .
\end{aligned}
$$

One can easily check that $\phi_{k}+\psi_{k}$ belongs to the Toda phase space for each positive integer $k$, and by construction $\psi_{k} \rightarrow 0$ as $k \rightarrow \infty$. The solution to the lift equation for $\phi_{k}$ is $f_{k}(t)=\exp \frac{t}{i} \phi_{k}$, and since $\frac{1}{i} \phi_{k}$ belongs to the algebra $\mathfrak{k}$ it follows that $f_{k}(t)$ lies in $\mathbf{K}$ for all $t$.

Since $E+F=\operatorname{Ad}_{g} H$ we have $\phi_{k}=\operatorname{Ad}_{g}(p(k) H)$, so that

$$
\begin{aligned}
\mathrm{M}\left(\phi_{k}\right)=f_{k}(2 \pi)=\exp \left(-2 \pi i \phi_{k}\right) & =g \exp \left(-2 \pi i \frac{1+2 c k}{2 c} H\right) g^{-1} \\
& =g \exp \left(-2 \pi i \frac{1}{2 c} H\right) \exp (2 \pi i H)^{-k} g^{-1}
\end{aligned}
$$

But $\exp \left(2 \pi i H_{\alpha}\right)=\mathrm{Id}$, (in the adjoint representation $H_{\alpha}$ is diagonal with integer entries), and so $\exp (2 \pi i H)=\mathrm{Id}$. One also has that for each positive root $\alpha$,

$$
\alpha\left(\frac{1}{2 c} H\right)=\frac{1}{c} \alpha\left(\Sigma \epsilon_{i}\right)=\frac{1}{c} \mathrm{ht}(\alpha) \in \mathbb{Q} \cap(0,1),
$$

where ht $(\alpha)$ denotes the height of $\alpha$, so that $\exp \left(-2 \pi i \frac{1}{2 c} H\right)$ is regular in G. This implies that we have regularity of the monodromy

$$
\mathrm{M}\left(\phi_{k}\right)=g \exp \left(-2 \pi i \frac{1}{2 c} H\right) g^{-1}=\mathrm{M}_{0} .
$$

Thus the requirements (1), (2) and (3) are satisfied, and the proof is complete. 


\section{The flows.}

In this section we prove that the period lattice of the Hamiltonian flows of the $F_{j}$ 's is trivial. We conclude that these functions form a completely integrable system and that this system is not the periodic Toda lattice.

Lemma 5.1. If $(\mu, 1)$ is a loop-regular element with $\mu \in \mathfrak{h}$, if $\gamma \in \widetilde{\mathrm{LG}}$ and if $\widetilde{\operatorname{Ad}}_{\gamma}^{*}(\mu, 1)=(\mu, 1)$, then $\gamma$ is a constant loop in $\mathbf{H}$.

Proof. Define $f(t)=\exp (-i \mu t)$. Note $f(t) \in \mathbf{H}$ for all $t$. Then $f$ solves the monodromy equation for $(\mu, 1)$ :

$$
\frac{d f(t)}{d t} f(t)^{-1}=\frac{1}{i} \mu .
$$

Since $\widetilde{\operatorname{Ad}}_{\gamma}^{*}(\mu, 1)=(\mu, 1)$, it follows that $\gamma\left(e^{i t}\right) f(t) \gamma(1)^{-1}$ also solves the monodromy equation for $(\mu, 1)$. So for all $t$ we have $\gamma\left(e^{i t}\right) f(t) \gamma(1)^{-1}=f(t)$. By the loop-regularity of $(\mu, 1), f(2 \pi)$ is regular in $\mathbf{H}$, so $\gamma(1) \in \mathbf{H}$.

Now for any $t$ we have $\gamma\left(e^{i t}\right)=f(t) \gamma(1) f(t)^{-1}=\gamma(1)$, since $f(t)$ and $\gamma(1)$ both lie in $\mathbf{H}$. So $\gamma$ is a constant loop in $\mathbf{H}$.

Lemma 5.2. Suppose $(\phi, 1)$ is a loop-regular element of the periodic Toda lattice phase space. Suppose $b \in \widetilde{\mathbf{B}}$ and

$$
\widetilde{\operatorname{Ad}}_{b}^{*}(\phi, 1)=(\phi, 1)
$$

Then $b=\mathrm{Id}$.

Proof. First, we claim that $b \in \tilde{\mathbf{N}}$. Write $b=a n$ with $a \in \mathbf{A}$ and $n \in \tilde{\mathbf{N}}$. We will show that $a=\mathrm{Id}$. Let ()$_{-1}$ denote the natural projection onto the height -1 subspace of $\widetilde{\mathrm{Lg}}$. (The algebra $\widetilde{\mathrm{Lg}}$ has a rootspace decomposition analogous to the finite-dimensional case and the notion of root height is well-defined, inducing a $\mathbb{Z}$-grading on $\widetilde{\mathrm{Lg}}$. See for example $[\mathbf{R S F}]$.) Then

$$
(\phi, 1)=\widetilde{\operatorname{Ad}}_{a}^{*} \widetilde{\operatorname{Ad}}_{n}^{*}(\phi, 1)
$$

and hence

$$
\begin{aligned}
(\phi, 1)_{-1} & =\widetilde{\operatorname{Ad}}_{a}^{*}\left(\widetilde{\operatorname{Ad}}_{n}^{*}(\phi, 1)\right)_{-1} \\
& =\widetilde{\operatorname{Ad}}_{a}^{*}(\phi, 1)_{-1} .
\end{aligned}
$$

Here the first equality holds because height is invariant under $\widetilde{\mathrm{Ad}}_{a}^{*}$. Since $\widetilde{\mathbf{N}}$ is generated by $\exp \tilde{\mathfrak{n}}$, and $\tilde{\mathfrak{n}}$ is the sum of rootspaces of positive height, 
we get the second equality after noting that Toda lattice elements have no component of height less than -1 .

However, because $(\phi, 1)$ is in the Toda lattice phase space we know that there exist strictly positive real numbers $q_{*}$ and $q_{i}, i=1, \ldots, \ell$ such that

$$
(\phi, 1)_{-1}=\left(\sum_{i} q_{i} E_{-\alpha_{i}}+z^{-1} q_{*} E_{\alpha_{*}}, 0\right) .
$$

Because $a \in \mathbf{A}$ we can write $a=\exp (X)$ for a unique $X \in \mathfrak{a}$. Then

$$
\widetilde{\operatorname{Ad}}_{a}^{*}(\phi, 1)_{-1}=\left(\sum_{i} e^{-\alpha_{i}(X)} q_{i} E_{-\alpha_{i}}+z^{-1} e^{\alpha_{*}(X)} q_{*} E_{\alpha_{*}}, 0\right) .
$$

Thus for each $\alpha \in \Pi$ we have $e^{\alpha(X)}=1$. Hence $X=0$ and $a=\mathrm{Id}$, so that $b \in \widetilde{\mathbf{N}}$. It follows that the constant term in the power series expansion of $b$ (which is just $b(0)$ ), lies in $\mathbf{N}$.

Since $(\phi, 1)$ is loop-regular, there is a $\mu \in \mathfrak{h}$ and a $\gamma \in \widetilde{\mathbf{K}}$ such that $(\phi, 1)=\widetilde{\operatorname{Ad}}_{\gamma}^{*}(\mu, 1)$ by Corollary 3.2. Hence

$$
\widetilde{\operatorname{Ad}}_{\gamma^{-1} b \gamma}^{*}(\mu, 1)=(\mu, 1),
$$

which implies that $\gamma^{-1} b \gamma \in \mathbf{H}$ by Lemma 5.1. Write $\eta=\gamma^{-1} b \gamma$. Then for any $z \in S^{1}$, we have $b(z)=\gamma(z) \eta \gamma(z)^{-1}$.

Using the realization of $\mathbf{G}$ as a linear subgroup of $S L(n, \mathbb{C})$ described in Section 2, now consider the characteristic polynomial $\operatorname{det}(b(z)-\lambda)$. Since $b(z) \in \widetilde{\mathbf{B}}$ this is an analytic function of $z$ on the open unit disk which is constant on the boundary of the disk since $\operatorname{det}(b(z)-\lambda)=\operatorname{det}(\eta-\lambda)$ for any $z \in S^{1}$. By an application of the maximum modulus principle it follows that this equality actually holds throughout the interior of the unit disk as well. In particular setting $z=0$ and using the fact that $b(0)$ lies in $\mathbf{N}$ gives

$$
\operatorname{det}(\eta-\lambda)=\operatorname{det}(b(0)-\lambda)=(1-\lambda)^{n} .
$$

So $\eta=\operatorname{Id}$ and $b(z)=\gamma(z) \operatorname{Id} \gamma(z)^{-1}=\operatorname{Id}$.

Proposition 5.1. Let $F_{1}, \ldots, F_{\ell}$ be the functions from Definition 3.2. Let $c_{1}, \ldots, c_{\ell}$ be real constants. Let $(\phi(t), 1)$ denote any trajectory of the Hamiltonian flow associated to $F=\sum_{j=1}^{\ell} c_{j} F_{j}$. If $(\phi(0), 1)$ is a loop-regular element of the periodic Toda phase space and $\phi(2 \pi)=\phi(0)$ then $c_{1}=c_{2}=$ $\cdots=c_{\ell}=0$. In other words, the period lattice for the real flows of the Hamiltonians $F_{1}, \ldots, F_{\ell}$ is trivial. 
Proof. By the factorization theorem $[\mathbf{R S}]$ the Hamiltonian flow associated to $F$ is given by

$$
(\phi(t), 1)=\widetilde{\operatorname{Ad}}_{b(t)}^{*}(\phi(0), 1),
$$

where $\exp [t \nabla F(\phi(0), 1)]=k(t)^{-1} b(t)$ with $k(t) \in \hat{\mathbf{K}}$ and $b(t) \in \hat{\mathbf{B}}$. If $\phi(2 \pi)=$ $\phi(0)$ then $(\phi(0), 1)=\widetilde{\operatorname{Ad}}_{b(2 \pi)}^{*}(\phi(0), 1)$, and by Lemma 5.2 it follows that $b(2 \pi)=$ Id. Hence $\exp [2 \pi \nabla F(\phi(0), 1)] \in \hat{\mathbf{K}}$.

However $\nabla F(\phi(0), 1) \in i \hat{\mathfrak{k}}$ by Corollary 3.3 , and so $\exp [2 \pi \nabla F(\phi(0), 1)]$ belongs to $\hat{\mathbf{K}} \cap \exp (i \hat{\mathfrak{k}})$. Because of the Cartan decomposition $\widetilde{\mathrm{LG}}=\hat{\mathbf{K}}$. $\exp (i \hat{\mathfrak{k}})$ this forces $\exp [2 \pi \nabla F(\phi(0), 1)]=\mathrm{Id}$, and $\nabla F(\phi(0), 1)=0$. Hence, also by Corollary $3.3, c_{1}=c_{2}=\cdots=c_{\ell}=0$ as required.

Notice that because of the decomposition $\widetilde{\mathrm{LG}}=\hat{\mathbf{K}} \hat{\mathbf{B}}$, by the factorization theorem the flows induced on the Toda phase space by the $F_{j}$ 's are complete. Note also that because $\mathrm{M}_{(\phi, 1)} \in \mathbf{K}$ for elements of the phase space, on the phase space we have

$$
F_{j}(\phi, 1)=\chi_{j}\left(\mathrm{M}_{(\phi, 1)}\right) .
$$

Corollary 5.1. The functions $F_{1}, \ldots, F_{\ell}$ constitute a completely integrable system on the Toda phase space.

Proof. It follows from the Kostant-Symes involution theorem that the functions $F_{1}, \ldots, F_{\ell}$ form a commuting family on the Toda phase space. Because the dimension of the Toda phase space is twice the number of functions, it remains to show that these functions are generically independent. By Proposition 4.1 it suffices to show that at any loop-regular element $(\phi, 1)$ the gradients of the restrictions of the functions $F_{1}, \ldots, F_{\ell}$ to the Toda phase space are linearly independent. But if these gradients were linearly dependent then $(\phi, 1)$ would be a fixed point of a nontrivial linear combination of the $F_{j}$ 's, which would contradict Proposition 5.1. Hence the functions are generically independent, and the system is completely integrable.

Corollary 5.2. The functions $F_{1}, \ldots, F_{\ell}$ do not all commute with the generalized real symmetric periodic Toda lattice Hamiltonian.

Proof. Pick a loop-regular point $(\phi, 1)$ in the phase space, and set $\vec{F}=$ $\left(F_{1}, \ldots, F_{\ell}\right)$. Let $\vec{F}_{c}$ denote the connected component of the level set of $\vec{F}$ containing $(\phi, 1)$ and note that this consists entirely of loop-regular elements. Because the flows induced by the $F_{j}$ 's commute and are complete there is an associated action of $\left(\mathbb{R}^{\ell},+\right)$ on $\vec{F}_{c}$. Since $\operatorname{dim} \vec{F}_{c}=\ell$ and the $F_{j}$ 's are functionally independent on the level set the orbits of this action are open in $\vec{F}_{c}$. But $\vec{F}_{c}$ is a disjoint union of such orbits and is connected, hence $\vec{F}_{c}$ 
consists of a single orbit and the action is transitive. Because the period lattice is trivial it follows that $\vec{F}_{c}$ is diffeomorphic to $\mathbb{R}^{\ell}$, and in particular is non-compact.

Now $\vec{F}_{c}$ is a closed subset of the Toda phase space, which itself can be regarded as a closed subset of $\mathbb{R}^{2 \ell+1}$ - essentially it is cut out by the polynomial equation $q_{*} \cdot \prod q_{i}^{k_{i}}=1$. Thus $\vec{F}_{c}$ is a closed non-compact subset of $\mathbb{R}^{2 \ell+1}$, and consequently is unbounded. But level sets of the Toda Hamiltonian $\mathrm{H}(\phi)=\mathrm{B}(\phi, \phi)$ are bounded and so the level set of $\mathrm{H}$ through $(\phi, 1)$ cannot contain the level set $\vec{F}_{c}$. Hence it cannot contain the flow of the $F_{j}$ 's through $(\phi, 1)$, and so $\mathrm{H}$ does not commute with all of the $F_{j}$ 's. 
Glossary of Notation

$\mathfrak{g}$......... a simple Lie algebra over $\mathbb{C}$

$\mathfrak{h}$.......... a Cartan subalgebra of $\mathfrak{g}$

$\mathfrak{b}$.......... a Borel subalgebra of $\mathfrak{g}$

$\mathfrak{k}$.......... a compact form of $\mathfrak{g}$

G ....... the simply connected Lie group corresponding to $\mathfrak{g}$

$\mathbf{H}$........ the Cartan subgroup of $\mathbf{G}$ corresponding to $\mathfrak{h}$

$\mathbf{B}$........ the Borel subgroup of $\mathbf{G}$ corresponding to $\mathfrak{b}$

$\mathbf{K}$........ the compact subgroup of $\mathbf{G}$ corresponding to $\mathfrak{k}$

$\mathrm{Lg}$....... the loop algebra

$\tilde{\mathfrak{b}}$.......... the algebra of loops with constant term in $\mathfrak{b}$, and no $z^{-1}$ terms

$\tilde{\mathfrak{k}}$......... the algebra of loops with image in $\mathfrak{k}$

LG ...... the loop group

$\widetilde{\mathbf{B}}$........ the subgroup of loops with constant term in $\mathbf{B}$, and no $z^{-1}$ terms

$\widetilde{\mathbf{K}}$......... the real subgroup of loops with image in $\mathbf{K}$

$\widetilde{\mathrm{Lg}}$........ the centrally extended loop algebra

$\hat{\mathfrak{b}}$......... the real subalgebra $\tilde{\mathfrak{b}}+\mathbb{R}$

$\hat{\mathfrak{k}} \ldots \ldots \ldots$ the real subalgebra $\tilde{\mathfrak{k}}+i \mathbb{R}$

$\widetilde{\mathrm{LG}} \ldots \ldots$ the centrally extended loop group

$\hat{\mathbf{B}}$......... the real subgroup corresponding to $\hat{\mathfrak{b}}$

$\hat{\mathbf{K}}$......... the real subgroup corresponding to $\hat{\mathfrak{k}}$ 


\section{References}

[AMR] R. Abraham, J.E. Marsden and T. Ratiu, Manifolds, Tensor Analysis and Applications, 2nd edition, Springer-Verlag, 1988.

[CL] E.A. Coddington and N. Levinson, Theory of Ordinary Differential Equations, McGraw-Hill Book Company, Inc., 1955.

[GW] R. Goodman and N.R. Wallach, Structure and unitary cocycle representations of loop groups and the group of diffeomorphisms of the circle, J. Reine Angew. Math., (1984), 69-133.

[GW2] Classical and quantum mechanical systems of Toda lattice type (II), Commun. Math. Phys., 94 (1984), 177-217.

[H] S. Helgason, Differential Geometry, Lie Groups and Symmetric Spaces, Academic Press, Inc., 1978.

[K] V.G. Kac, Infinite Dimensional Lie Algebras, 3rd edition, Cambridge University Press, 1990.

[Kn] A. Knapp, Representation Theory of Semisimple Groups; An Overview Based On Examples, Princeton University Press, 1986.

[Ko] B. Kostant, The solution to a generalized Toda lattice and representation theory, Adv. Math., 39 (1979), 195-338.

[L] S. Lang, Real Analysis, 2nd edition, Addison-Wesley, 1983.

[Pe] A.M. Perelomov, Integrable Systems of Classical Mechanics and Lie Algebras, Vol. I, Birkhäuser, 1990.

[PS] A. Pressley and G. Segal, Loop Groups, Clarendon Press, Oxford, 1988.

[Qu] M. Quinn, A new completely integrable system on the Toda lattice phase space, Ph. D. thesis, MIT, 1995.

[RS] A.G. Reyman and M.A. Semenov-Tian-Shansky, Group-theoretical methods in the theory of finite-dimensional integrable systems, Dynamical Systems VII, ed.'s V.I. Arnol'd and S.P. Novikov, Springer-Verlag, Berline, 1987.

[RSF] A.G. Reiman, M.A. Semenov-Tian-Shansky and I.E. Frenkel, Graded Lie Algebras and Completely Integrable Dynamical Systems, Soviet Math. Dokl., 20 (1979), 811814 .

[St] R. Steinberg, Conjugacy Classes in Algebraic Groups, Lecture Notes in Mathematics, 366 (1974), Springer-Verlag.

[Sy] W. Symes, Systems of Toda type, inverse spectral problems and representation theory, Invent. Math., 59 (1980), 13-53.

Received May 29, 1996 and revised October 19, 1996.

HAVERFord COLlege

HAVERFORD, PA 19041

E-mail address: ssinger@haverford.edu 